\title{
A Study on Risk Factors of Recurrent Pregnancy Loss
}

\author{
Dr. Dorothy Shahnaz Mukul Fatema ${ }^{1 *}$, Dr. Abdul Khaleque ${ }^{2}$, Dr. Salma Rouf ${ }^{3}$
}

${ }^{1}$ Department of Gynaecology and Obstetrics, Dhaka Medical College Hospital, Bangladesh

${ }^{2}$ Department of Orthopaedics, Dhaka Medical College Hospital, Bangladesh

${ }^{3}$ Professor, Department of Gynaecology and Obstetrics, Dhaka Medical College Hospital, Bangladesh

DOI: $10.36348 /$ sijog.2021.v04i01.001

| Received: 12.11 .2020 | Accepted: 23.11.2020 | Published: 08.01.2021

*Corresponding author: Dr. Dorothy Shahnaz Mukul Fatema

\section{Abstract}

Recurrent pregnancy loss (RPL) is a condition when a woman has three or more consecutive pregnancy loss. A loss of pregnancy is described as a clinically recognized pregnancy that ends involuntarily before 20 weeks. Most complications in pregnancy arise from chromosomal, or genetic, defects and are spontaneous occurrences. The main aim of this research is to find out the risk factors associated with Recurrent Pregnancy Loss (RPL). Also, the specific objectives of the study are; to recognize the probable remedial targets to detect the origin of the pregnancy losses, and to provide necessary recommendations regarding organized care for couples with RPL. A descriptive study was conducted for 1 year from July 2014 to June 2015, to find out the cause and consequences of recurrent pregnancy loss. Factors, such as age, environmental and occupational exposure, psychological pressure, chronic endometritis, genetic factors, smoking, thrombophilia screening, uterine imperfections, were some of the risks for Recurrent Pregnancy Loss. Multiple miscarriages can create noteworthy negative mental pressure for couples, and that is why numerous studies are being conducted to improve medicines and reduce the time expected to accomplish an effective and full-term pregnancy. Patients with RPL need to consult with fertility specialists about appropriate exams to clarify the risk factors and treatments to improve the outcomes of pregnancy.

Keywords: Recurrent Miscarriage, RPL, Chronic Endometritis, Bangladeshi people.

Copyright () 2021 The Author(s): This is an open-access article distributed under the terms of the Creative Commons Attribution 4.0 International License (CC BY-NC 4.0) which permits unrestricted use, distribution, and reproduction in any medium for non-commercial use provided the original author and source are credited.

\section{INTRODUCTION}

Recurrent Pregnancy Loss (RPL) is depicted as at least three consecutive loss of pregnancy and this upsetting issue impacts around $1 \%$ everything being equivalent. Although about $15 \%$ of all clinically recognized pregnancies end in spontaneous miscarriage, there are many more unconstrained untimely births are tedious miscarriage $[1,2]$. Some studies claim that two as not three pregnancy losses are adequate to represent RPL.

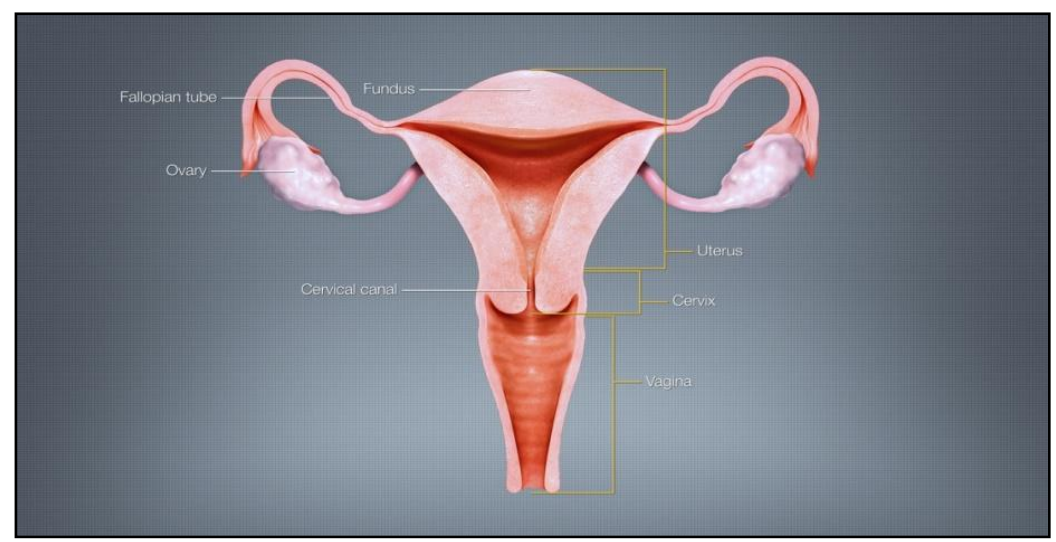

Fig-1: Uterus Anatomy

Citation: Dorothy Shahnaz Mukul Fatema et al (2021). A Study on Risk Factors of Recurrent Pregnancy Loss. Sch Int J Obstet Gynec, 4(1), 1-5. 
A pregnancy loss (unexpected labor/unsuccessful labor) is described as the unconstrained destruction of a pregnancy before the fetus shows up at reasonability. The term thusly consolidates all pregnancy losses from the hour of beginning until 24 weeks of development. It should be seen that progress in neonatal thought has achieved scarcely any babies suffering birth before 24 weeks of growth and different definitions apply in different countries [3].

By definition, "recurrent" PL is portrayed as the loss of two pregnancies by any event. In any case, to which degree this definition ought to be expanded or stifled is less clear, as is showed up by changed definitions used in different standards and different countries.

\section{OBJECTIVE}

The main aim of this study is to determine risk factors of Recurrent Pregnancy Loss (RPL) among the patients.

\section{Scope}

The general purpose of this article is to provide restorative administration providers with the best available evidence for the assessment and treatment of females with RPL. RPL is described as the loss of fetuses in any event during two pregnancies. It keeps away from an ectopic pregnancy and molar pregnancy. The article covers the thought gave by discretionary and tertiary human administration specialists who have direct contact with, and choose decisions concerning the thought of, couples with repetitive pregnancy misfortune or unnatural birth cycles. To give barely any directions and sharedessential initiative, a patient adjustment of this article will be made.

\section{Study Procedure}

A descriptive study was conducted for 1year; from July 2014 to June 2015, to find out the cause and consequences of recurrent pregnancy loss.

\section{DISCUSSION}

\section{Risk Factors of RPL}

\section{Psychological Pressure}

Maternal stress during and after pregnancy is possibly associated with an extended risk of a couple of ominous pregnancy and birth results, anyway, there are correct now no brilliant investigations open. In our examination, the impact of weight on the risk of unexpected labor or redundant pregnancy misfortune is not so apparent.

In any case, we found two investigations reviewing worry in ladies with RPL. From a casecontrol study, it was observed that weight is a peril factor for RPL reliant on the finding among 45 ladies with unexplained RPL differentiated and 40 controls [4]. In another examination, stress and misery were assessed in 301 RPL patients and 1813 ladies without RPL endeavoring to consider. A high tension, described as $\geq 19$ on the PSS scale, was progressively unavoidable in ladies with RPL (41.2\%) when appeared differently about controls (23.2\%). Similarly, the odds of moderate to outrageous wretchedness was an abundance of different occasions higher in ladies with RPL [5]. One little examination (25 pregnancies) on pregnancy misfortune and worry during pregnancy demonstrated a connection between maternal weight and pregnancy misfortune, maybe intervened through higher cortisol levels [6].

Age

Female age is a huge risk factor for RPL; women that are over 40 years have a higher threat of RPL and have increasingly lamentable expectations diverged from progressively energetic ladies. In couples resolved to have RPL, the information that age is a risk factor is up 'til now noteworthy as it may impact the indicative frameworks and the essential initiative of treatment or confident organization. A connection between menstrual age and RPL has been dependably showing up in a couple of examinations. Cauchi and partners assumed that females age under 30 years arerelated to a very basic level of progress rate in resulting pregnancy in ladies with RPL and the age above 30 years is a risk factor for pregnancy misfortune in ladies with $\mathrm{RPL}^{7}$.An expressive associate investigation assessing the chance of live birth in 987 RPL couples during a 5-year follow-up period found an imperative diminishing in the chance of live birth with a growing female age [8].

In an associate investigation investigating factors related to PL in 696 ladies with RPL, and a female age $\geq 35$ years was found to two-fold the threat of another PL, stood out from ladies < 35 years (OR 1.99; 95\% CI 1.45-2.73) [9]. Our examination derived that the female age over 35 years was the immense marker of the chromosomal idiosyncrasy peril in RPL.

\section{Smoking}

Smoking is unequivocally associated with adversarial obstetric and neonatal outcomes, including ectopic pregnancy, stillbirth, placenta praevia, preterm birth, low birth weight, and other natural anomalies. Studies have showna connection between maternal smoking during pregnancy and issues during youth, including unforeseen child passing issues, weight, psychosocial issues, and malignancies [10].

Regardless, we found the impact of smoking or smoking suspension on pregnancy misfortune in ladies with RPL is less clear. Various examinations have surveyed the effect of maternal and protective smoking on the risk of irregular pregnancy misfortune. We found that the impact of male smoking was 
Dorothy Shahnaz Mukul Fatema et al; Sch Int J Obstet Gynec, Jan. 2021; 4(1): 1-5

progressively basic in significant smokers who appeared differently about direct smokers.

\section{Environmental and Occupational Exposure}

We discovered just two examinations evaluating word related or common introduction as a risk factor for RPL. In the primary examination serum; zinc, copper, and supplement E levels were lower in 35 ladies with RPL, and serum selenium, lead, and cadmium were on a basic level, which could show that significant metals and nonattendance of micronutrients could cause pregnancy misfortune in ladies with RPL [11]. In the ensuing examination, progressively critical degrees of organochlorine pesticides were perceived in the blood of 30 ladies with RPL appeared differently about 30 controls, which could exhibit a connection between organochlorine pesticides and RPL [12]. Our investigations give an equivalent circumstance among a bit of the maternity patients of the clinical center. Regardless, it was a test to perceive the exact normal or conceivably word related condition of said patients.

Taking into account only a few little examinations, prologue to word related and characteristic parts are related with an extended peril of pregnancy misfortune in ladies with RPL.

\section{Chronic Endometritis}

Interminable/Chronic Endometritis (CE) is portrayed as incessant bothering of the endometrial covering, and a couple of studies have shown extended normality in ladies with RPL (10\%-27\%) [13-15]. Endometrial receptivity is accepted to be hindered by the stromal intrusion of plasma cells, similarly, as adjusted verbalizations of characteristics drew in with implantation, inciting RPL yet likewise subfertility and monotonous implantation dissatisfaction following in vitro planning (IVF). A few methodologies have been used to assert the examination of $\mathrm{CE}$, yet the best quality level is recognizing the verification of plasma cells in the endometrial stroma. There are no investigations differentiating paces of endometritis in ladies and RPL to control ladies, or discussing the perceptive estimation of a positive test for endometritis.

In any case, we found that disease prevention administrators can eliminate the endometritis with an obvious improvement in the live birth rate. This idea has not endeavored in randomized controlled basics.

\section{Genetic Factors}

Hereditary or inherited factors from the standard of the conceptus are an apparent purpose behind irregular and tedious pregnancy misfortune (RPL). In an exact study, the regularity of chromosome abnormalities in a singular irregular unnatural birth cycle was $45 \%$ (95\% CI 38-52; 13 examinations; 7012 models). The ordinariness of chromosome anomalies in the following fruitless work resulting in going before RPL was indistinguishable [16]. It is possible to see if an early pregnancy misfortune is a direct result of an innately sporadic lacking life form or undeveloped organism by analyzing the pregnancy or fetal tissue [17].

In certain cases, there is a disappointment in ordinary control instruments that forestall an invulnerable response against self, bringing about an immune system reaction. Autoantibodies to phospholipids, thyroid antigens, atomic antigens, and others have been researched as potential foundations for pregnancy misfortune. Antiphospholipid (aPL) antibodies incorporate the lupus anticoagulant, Antibeta 2 glycoprotein I antibodies, and anticardiolipin antibodies. An expanding number of studies recommend that antibodies to phosphatidylserine are likewise connected with pregnancy misfortune.

In our assessment, choosing the chromosomal status of pregnancy tissue from ladies with repetitive pregnancy misfortune outfitted them with an explanation or reason behind the particular misfortune being investigated, yet it didn't generally block other concealed conditions.

\section{Thrombophilia}

Thrombophilia by definition speaks to obtained as well as hereditary conditions which incline patients to both venous and blood vessel thromboembolic occasions. Apoplexy is the most widely recognized reason for death around the world. On the blood vessel side, myocardial dead tissue and stroke bring about critical horribleness and mortality. Pregnancy is a hypercoagulable state, and thromboembolism is the main source of antepartum and maternal mortality. Late consideration has concentrated on certain acquired thrombophilic factors that may incline to blood vessels as well as venous apoplexies and their conceivable relationship with pregnancy entanglements, including early pregnancy misfortune. These incorporate a gathering of generally autosomal prevailing, acquired quality transformations prompting a hypercoagulable state. Current comprehension demonstrated that a mix of hazard factors, including different acquired thrombophilic deserts related to optional hypercoagulable states, (for example, pregnancy and antiphospholipid syndrome) can be related to unfavorable pregnancy result.

\section{Uterine Defects}

We considered the defect as a noteworthy explanation behind RPL. Uterine irregularities are supposedly found in up to $19 \%$ of ladies with RPL and can be named gotten or intrinsic [18].

Picked up anomalies fuse intrauterine connections, myomas, and endometrial polyps. Intrauterine connections, or synechiae, occur in regions where the endometrial basal layer has been crushed, generally a significant part of the time following 
Dorothy Shahnaz Mukul Fatema et al; Sch Int J Obstet Gynec, Jan. 2021; 4(1): 1-5

curettage, a uterine clinical system, or infection, or a snared birth [19]. The repeat and reality of bonds increase with the amount of curettages [19]. Submucosalmyomas are found in $4.5 \%$ of ladies with RPL and should be correctly cleared at whatever point diagnosed [20]. Polyps are found in $2 \%-3 \%$ of ladies with RPL and should be hysteroscopically resected [21]. Cervical uncouthness, as a rule, causes secondtrimester misfortune, and it will in general be acquired after cautious injury or is connected with inborn uterine abnormalities [21].

\section{CONCLUSION AND RECOMMENDATION}

The long-haul anticipation of couples with repeated loss of pregnancy is fantastic, and most eventually achieve a sound live birth. Be that as it may, specific pregnancy losses for affected couples that have a substantial mental cost, and various attempts are being made to develop drugs and minimize the time required for a successful pregnancy. In this way, it is suggested that; women should be sensitively educated that in women aged 20 to 35 years, the hazard regarding pregnancy loss is most minimal. Stress is related to RPL but couples should be educated that there is no evidence that pressure is an immediate reason for the loss of pregnancy. Although RPL diagnosis can be quite devastating, keeping in mind the relatively high likelihood that the next pregnancy will be successful can be helpful for the physician and patient. The prognosis of a particular individual will depend on both the underlying cause of loss of pregnancy and the number of preceding losses. Patients with RPL need to consult with fertility specialists about appropriate exams to clarify the risk factors and treatments to improve the outcomes of pregnancy.

\section{REFERENCES}

1. Daya, S. (1996). Evaluation and management of recurrent spontaneous abortion. Current Opinion in Obstetrics and Gynecology, 8(3), 188-192.

2. Sierra, S., \& Stephenson, M. (2006, February). Genetics of recurrent pregnancy loss. In Seminars in reproductive medicine $(\mathrm{Vol}$. 24, No. 01, pp. 017-024). Copyright@ 2006 by Thieme Medical Publishers, Inc., 333 Seventh Avenue, New York, NY 10001, USA..

3. Green-top Guideline. (2011). The investigation and treatment of couples with recurrent firsttrimester and second-trimester miscarriage.

4. Li, W., Newell-Price, J., Jones, G. L., Ledger, W. L., \& Li, T. C. (2012). Relationship between psychological stress and recurrent miscarriage. Reproductive biomedicine online, 25(2), 180-189.

5. Kolte, A. M., Olsen, L. R., Mikkelsen, E. M., Christiansen, O. B., \& Nielsen, H. S. (2015). Depression and emotional stress is highly prevalent among women with recurrent pregnancy loss. Human reproduction, 30(4), 777-782.
6. Nepomnaschy, P. A., Welch, K. B., McConnell, D. S., Low, B. S., Strassmann, B. I., \& England, B. G. (2006). Cortisol levels and very early pregnancy loss in humans. Proceedings of the National Academy of Sciences, 103(10), 39383942.

7. Cauchi, M. N., Pepperell, R., Kloss, M., \& Lim, D. (1991). Predictors of pregnancy success in repeated miscarriage. American Journal of Reproductive Immunology, 26(2), 72-75.

8. Lund, M., Kamper-Jørgensen, M., Nielsen, H. S., Lidegaard, Ø., Andersen, A. M. N., \& Christiansen, O. B. (2012). Prognosis for live birth in women with recurrent miscarriage: what is the best measure of success?. Obstetrics \& Gynecology, 119(1), 37-43.

9. Lo, W., Rai, R., Hameed, A., Brailsford, S. R., AlGhamdi, A. A., \& Regan, L. (2012). The effect of body mass index on the outcome of pregnancy in women with recurrent miscarriage. Journal of family \& community medicine, 19(3), 167-171.

10. Leung, L. W., \& Davies, G. A. (2015). Smoking cessation strategies in pregnancy. Journal of Obstetrics and Gynaecology Canada, 37(9), 791797.

11. Ajayi, O. O., Charles-Davies, M. A., \& Arinola, O. G. (2012). Progesterone, selected heavy metals and micronutrients in pregnant Nigerian women with a history of recurrent spontaneous abortion. African health sciences, 12(2), 153-159.

12. Pathak, R., Mustafa, M. D., Ahmed, R. S., Tripathi, A. K., Guleria, K., \& Banerjee, B. D. (2010). Association between recurrent miscarriages and organochlorine pesticide levels. Clinical biochemistry, 43(1-2), 131-135.

13. Kitaya, K. (2011). Prevalence of chronic endometritis in recurrent miscarriages. Fertility and sterility, 95(3), 1156-1158.

14. McQueen, D. B., Perfetto, C. O., Hazard, F. K., \& Lathi, R. B. (2015). Pregnancy outcomes in women with chronic endometritis and recurrent pregnancy loss. Fertility and sterility, 104(4), 927 931.

15. Bouet, P. E., El Hachem, H., Monceau, E., Gariépy, G., Kadoch, I. J., \& Sylvestre, C. (2016). Chronic endometritis in women with recurrent pregnancy loss and recurrent implantation failure: prevalence and role of office hysteroscopy and immunohistochemistry in diagnosis. Fertility and sterility, 105(1), 106-110.

16. Van den Berg, M. M. J., Vissenberg, R., \& Goddijn, M. (2014). Recurrent miscarriage clinics. Obstetrics and Gynecology Clinics, 41(1), 145-155.

17. Mathur, N., Triplett, L., \& Stephenson, M. D. (2014). Miscarriage chromosome testing: utility of comparative genomic hybridization with reflex microsatellite analysis in preserved miscarriage tissue. Fertility and sterility, 101(5), 1349-1352. 
18. Jaslow, C. R., Carney, J. L., \& Kutteh, W. H. (2010). Diagnostic factors identified in 1020 women with two versus three or more recurrent pregnancy losses. Fertility and sterility, 93(4), 1234-1243.

19. Deans, R., \& Abbott, J. (2010). Review of intrauterine adhesions. Journal of Minimally Invasive Gynecology, 17(5), 555-569.
20. Jaslow, C. R. (2014). Uterine factors. Obstetrics and Gynecology Clinics, 41(1), 57-86.

21. Salim, S., Won, H., Nesbitt-Hawes, E., Campbell, N., \& Abbott, J. (2011). Diagnosis and management of endometrial polyps: a critical review of the literature. Journal of minimally invasive gynecology, 18(5), 569-581. 